\title{
La desigual distribución de la desigualdad. Política subnacional y distribución del ingreso en las provincias argentinas, 2003-2011*
}

The Unequal Distribution of Inequality. Subnational Politics

and Income Distribution in the Argentine Provinces, 2003-2011

\section{LUCAS GONZÁLEZ}

Consejo Nacional de Investigaciones Científicas y Técnicas, Argentina

Universidad Nacional de San Martín, Argentina

Universidad Católica Argentina, Argentina

lgonzalez@unsam.edu.ar; lucas_gonzalez@uca.edu.ar

\section{MARCELO NAZARENO}

Universidad Católica de Córdoba-Unidad Asociada al Consejo Nacional de Investigaciones Científicas y Técnicas, Argentina

Universidad Nacional de Córdoba, marcelonazareno@arnet.com.ar

En América Latina, a pesar de las mejoras en los niveles de desigualdad a nivel nacional, la desigualdad es aún elevada y hay indicios de que el proceso de su disminución se ha detenido. Una de las explicaciones de los limites en la reducción de la desigualdad debe buscarse en el nivel subnacional, ya que en este nivel perviven bolsones de muy elevada desigualdad aun cuando los gobiernos nacionales apliquen politicas redistributivas. En este trabajo indagamos sobre los factores en este nivel de gobierno que contribuyan a explicar las diferencias en la desigualdad de ingresos entre las provincias de la Argentina para ayudar a comprender los alcances y los límites de los procesos nacionales de reducción de la desigualdad. Luego de describir los casos usando datos de panel inéditos de desigualdad de ingreso y revisar la literatura sobre países de la región, el trabajo avanza en la identificación de factores políticos relacionados sistemáticamente con los niveles de desigualdad de las provincias argentinas.

\section{Introducción}

En los federalismos latinoamericanos, en general, y la Argentina, en particular, la desigualdad de ingresos está desigualmente distribuida. En

Articulo aceptado para su publicación el 30 de mayo de 2019. 
efecto, la variación de los niveles de desigualdad considerada dentro de las diferentes unidades subnacionales es enorme. En el caso argentino, Tierra del Fuego es muy igualitaria para los estándares latinoamericanos, ya que tiene un índice de Gini provincial de 0,32 (en 2011), similar a Canadá y Australia (en 2011 y 2012, respectivamente. WIID, 2017). Pero Salta y Corrientes, por el contrario, son las provincias más desiguales de Argentina, con índices de Gini de 0,45 y 0,46 (en 2011) respectivamente, similares a Guatemala, Malawi y Paraguay (2014, 2010 y 2013 respectivamente; SEDLAC, 2016, WIID, 2017) $)^{1}$.

Además, el «ritmo» de variación de la desigualdad es también muy distinto entre las diferentes provincias argentinas. Si analizamos la variación del índice de Gini en las provincias entre 2003 y 2011 podemos observar una caída importante de la desigualdad. El Gini promedio para el primer año de la serie es 0,50; para el último año de la serie, la media es 0,40 (diez puntos promedio menos). Sin embargo, en el marco de esta caída en promedio de la desigualdad, subyacen fuertes diferencias entre las distintas provincias. Salta y Chaco lograron disminuirla mucho durante los 2000. Salta tuvo la mayor desigualdad del país en el primer año de la serie (0,59 en 2003), bajando su índice en casi 15 puntos (llegando a 0,45 en 2011). Chaco, de manera similar, pasó de 0,52 a 0,36. Por el contrario, Corrientes y Jujuy, siendo bastante desiguales, disminuyeron menos su desigualdad (la primera pasó de 0,56 a 0,45 y la segunda de 0,50 a 0,42). Por otra parte, Tierra del Fuego, siendo relativamente igualitaria durante toda la serie, redujo su desigualdad en 10 puntos, convirtiéndose en la provincia menos desigual del país para el último año de la serie (González y Nazareno, 2018).

En este artículo abordaremos los factores políticos que pueden explicar esta «desigual distribución de la desigualdad interna» entre las provincias argentinas y su diferente «ritmo» de cambio. Nos parece razonable pensar que esta gran variación, con provincias mostrando niveles de desigualdad muy altos y/o que en contextos nacionales de reducción de desigualdad consiguen reducirla muy poco o de modo insuficiente, es un elemento que incide para que la desigualdad nacional, a pesar de su fuerte reducción en los últimos años, parezca tener un umbral aún demasiado alto que es difícil de perforar a través de políticas nacionales. Nuestro interés se centra en identificar los factores políticos que, operando en el nivel de los gobiernos subnacionales, contribuyan a explicar esta variación en-

Los estados de Brasil son también presentan un rango amplio en cuanto a su desigualdad interna, con una distribución que va desde estados relativamente igualitarios (Santa Catarina tiene un Gini de 0,44 en 2013) a otros muy desiguales (como Brasilia, que tiene un Gini de 0,58 en 2013) (IPEADATA, 2017). 
tre las provincias y que podrían fijar un límite a la reducción de la desigualdad a nivel nacional ${ }^{2}$.

Creemos que entender esta dinámica subnacional es muy relevante en el actual contexto latinoamericano. Es muy conocido que en América Latina la desigualdad es profunda y endémica. Luego de un agravamiento de la situación durante los 80 s y 90 s del siglo pasado, la desigualdad de ingresos en la región (incluyendo a la Argentina) mostró tendencias notoriamente decrecientes desde comienzos del nuevo siglo (Birdsall, Lustig y McLeod 2011; Blofield, 2011; Gasparini y Lustig, 2011; Cornia, 2012; Lustig et al. 2013). Sin embargo, muchos observadores señalaron y señalan los límites de este progreso: no sólo la desigualdad en la región y en nuestro país sigue siendo muy alta, sino que se abren dudas respecto de una continuidad significativa en su descenso, en la medida que se observan condiciones y mecanismos económicos y sociales que comenzaron a operar, o se espera que pronto comiencen a hacerlo, en un sentido inverso ${ }^{3}$.

También inciden, ciertamente, circunstancias políticas. Más allá del papel central que juega el signo político de los gobiernos nacionales, algunos autores señalan factores políticos que operan aún en el marco de gobiernos nacionales con predisposición a implementar políticas de redistribución progresiva (Blofield, 2011; Beramendi, Rogers y Díaz-Cayeros, 2017).

Uno de estos factores políticos es el modo en que (sobre todo, pero no únicamente, en los sistemas federales) la dinámica política en los niveles subnacionales condiciona, diluye e incluso bloquea las iniciativas de reducción de la desigualdad que puedan tomarse en el nivel nacional. Nuestro interés apunta a identificar, para el caso argentino, las variables que, operando en el nivel político específicamente subnacional, establecen un límite a la reducción de la desigualdad en este nivel y, con ello, afectan también las tendencias nacionales.

Nuestra pretensión es abrir una línea de indagación que creemos muy relevante y que aún no muestra signos de desarrollo consolidado respecto

2 Una línea de indagación diferente, pero complementaria de la que desarrollaremos en este proyecto, es la que plantean Beramendi, Rogers y Díaz-Cayeros (2017) quienes se centran, en lugar de los factores políticos subnacionales que influyen sobre las desigualdades subnacionales, en la incidencia política que los sectores económicos y políticos con base subnacional tienen sobre la política y sobre los niveles de desigualdad nacionales.

3 La CEPAL advertía recientemente sobre el estancamiento del proceso de reducción de la desigualdad en la región, con índices de Gini en 2016 similares a los que se había alcanzado en 2014 tras varios años de un descenso relativamente rápido (CEPAL 2017: 14). En cuanto a las razones socio-económicas de este estancamiento, Lustig, López Calva y Ortiz Juarez (2011) marcan los problemas derivados de la calidad educativa en los sectores de más bajos ingresos mientras Wibbels (2015) por su parte, señala los límites a la redistribución que imponen las características de los mercados laborales latinoamericanos. 
de la Argentina y América Latina en general (más allá de unos pocos trabajos a los que nos referiremos más adelante). En este sentido, puede considerarse que se trata de un trabajo en buena medida exploratorio (tanto en términos teórico-conceptuales como metodológicos y empíricos) orientado a establecer algunas bases para futuras indagaciones.

Está organizado del siguiente modo. En la siguiente sección ofrecemos algunos datos sistematizados que dan un panorama de la evolución y las diferencias en los niveles de desigualdad en las provincias argentinas. Luego nos ocupamos de algunos aspectos teóricos y conceptuales con intención de sistematizar los desarrollos de la literatura pertinente e identificar los vacíos analíticos aún existentes. En la tercera sección establecemos algunas correlaciones significativas entre variables relevantes y los niveles de desigualdad en las provincias de la Argentina. Finalmente, extraemos algunas conclusiones e intentamos establecer una agenda de futuras indagaciones.

\section{La Desigual Distribución de la Desigualdad en las Provincias Argentinas}

La desigualdad al interior de las provincias argentinas muestra grandes diferencias tanto en sus valores absolutos como en su evolución a lo largo del tiempo (más allá de una generalizada tendencia decreciente durante el período analizado $)^{4}$. Los datos muestran que la provincia más desigual de la Argentina en el último año de la serie es Corrientes ${ }^{5}$. Su índice de Gini para 2011 es 0,46, seguida por Salta $(0,45)$. En el otro extremo de la distribución, las provincias más igualitarias son Tierra del Fuego y Formosa (con 0,32 y 0,34 en 2011, respectivamente). El Gráfico 1 muestra la variación de Gini de ingresos en las provincias argentinas entre 2003 y 2011.

4 La desigualdad es un fenómeno multidimensional que involucra múltiples "tipos" de desigualdades que se diferencian entre sí tanto respecto a qué refiere la desigualdad (educación, salud, vivienda, etc.), como entre quienes la desigualdad se conceptualiza y se mide (McKey: 1-2). En este trabajo nos focalizamos en el análisis de la desigualdad interpersonal de ingresos (después de impuestos y transferencias). Esperamos en trabajos posteriores abordar otras dimensiones de la desigualdad dentro de las provincias argentinas.

$5 \quad$ En este trabajo usamos los datos del índice de Gini provinciales calculados por Calvo y Moscovich (2017: 11) entre 2003 y 2011. Para ello, los autores usaron datos de encuestas nacionales (Encuesta Permanente de Hogares, EPH) del Instituto Nacional de Estadísticas y Censos (INDEC) para las 32 grandes regiones metropolitanas del país. Cada encuesta incluye entre 45.000 y 65.000 entrevistados, para un total de 1.838 .828 individuos. Un total de 968.833 recibió algún ingreso en el momento de la encuesta. Aunque las encuestas de ingresos nacionales se han aplicado desde 1974, los autores agruparon solamente un subconjunto estandarizado que incluye un grupo idéntico de 


\section{Gráfico 1}

Desigualdad Interpersonal en las Provincias Argentinas (2003-2011)

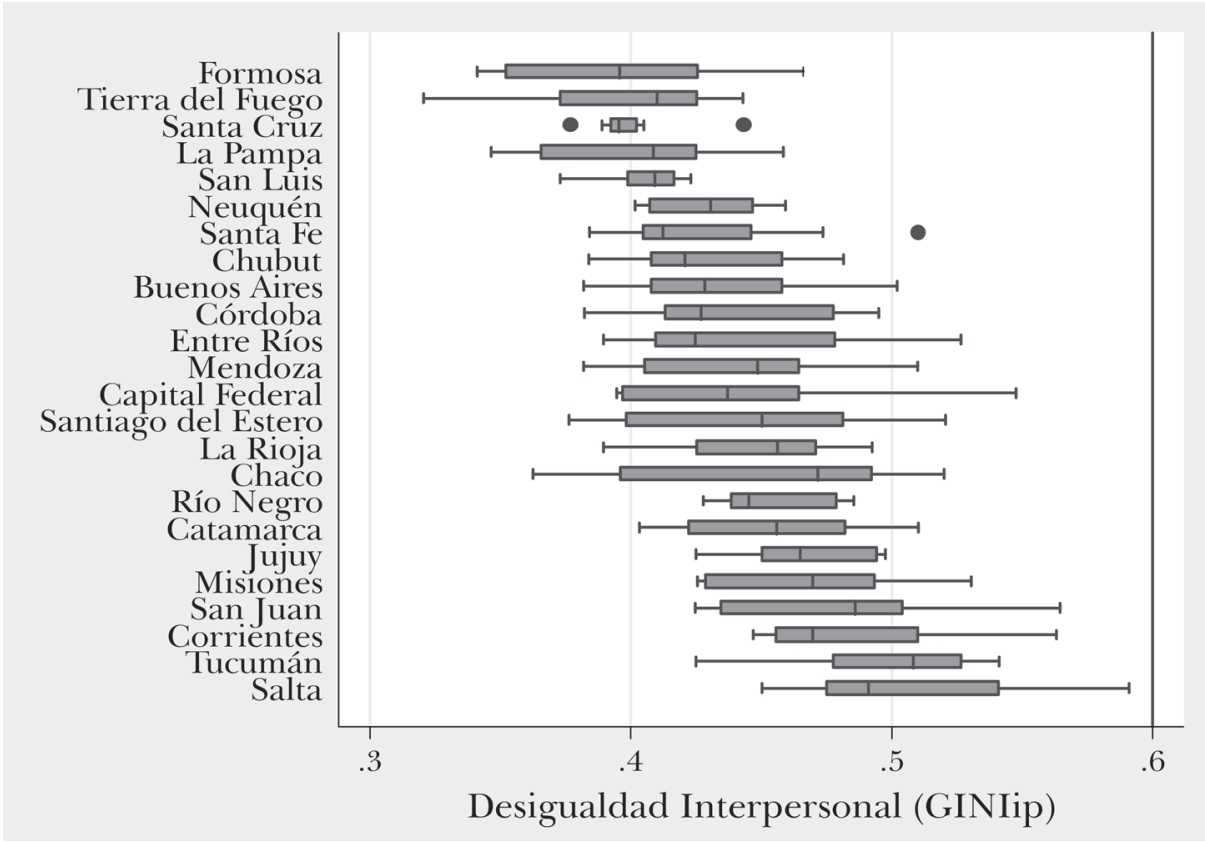

Fuente: elaboración propia en base a datos de EPH de Calvo y Moscovich (2017).

En el marco de esta caída en promedio de la desigualdad, se observan fuertes diferencias entre las distintas provincias. En la introducción resaltamos los casos de Salta y Chaco, que lograron disminuirla mucho, y de Corrientes y Jujuy que disminuyeron menos su desigualdad (ver Gráficos 2 y 3).

preguntas y variables. Al tratarse de una encuesta aplicada a poblaciones de centros urbanos (capitales provinciales y aquellos de más de 100 mil habitantes) los índices de Gini calculados en base a la EPH no corresponden a la totalidad de la población provincial, pudiendo haber diferencias significativas entre ambos. No hay datos alternativos que permitan subsanar esta dificultad. Si bien por cuestiones de comodidad expositiva seguimos llamando en lo que sigue a estos índices de Gini "índices provinciales", enfatizamos que los resultados de los análisis que obtenemos a partir de ellos deben tomarse con precaución dada esta característica segmentada y urbana de la población a la que se refieren. 
Revista SAAP $\cdot$ Vol. 13, № 1

Gráfico 2

Cambios en la Desigualdad en las Provincias Argentinas (2003-2011)

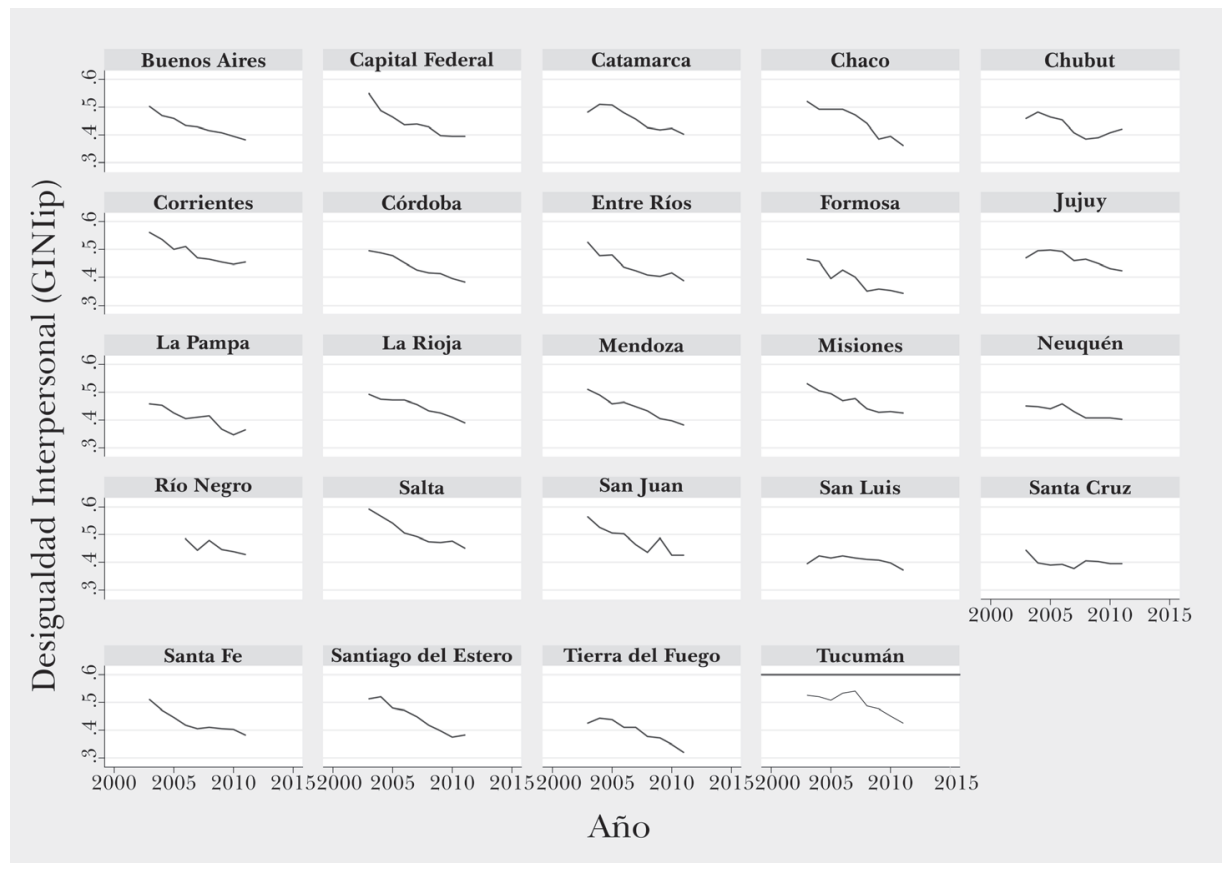

Fuente: elaboración propia en base a datos de EPH de Calvo y Moscovich (2017). 


\section{Gráfico 3}

Cambios en la Desigualdad en las Provincias Argentinas (2003-2011)

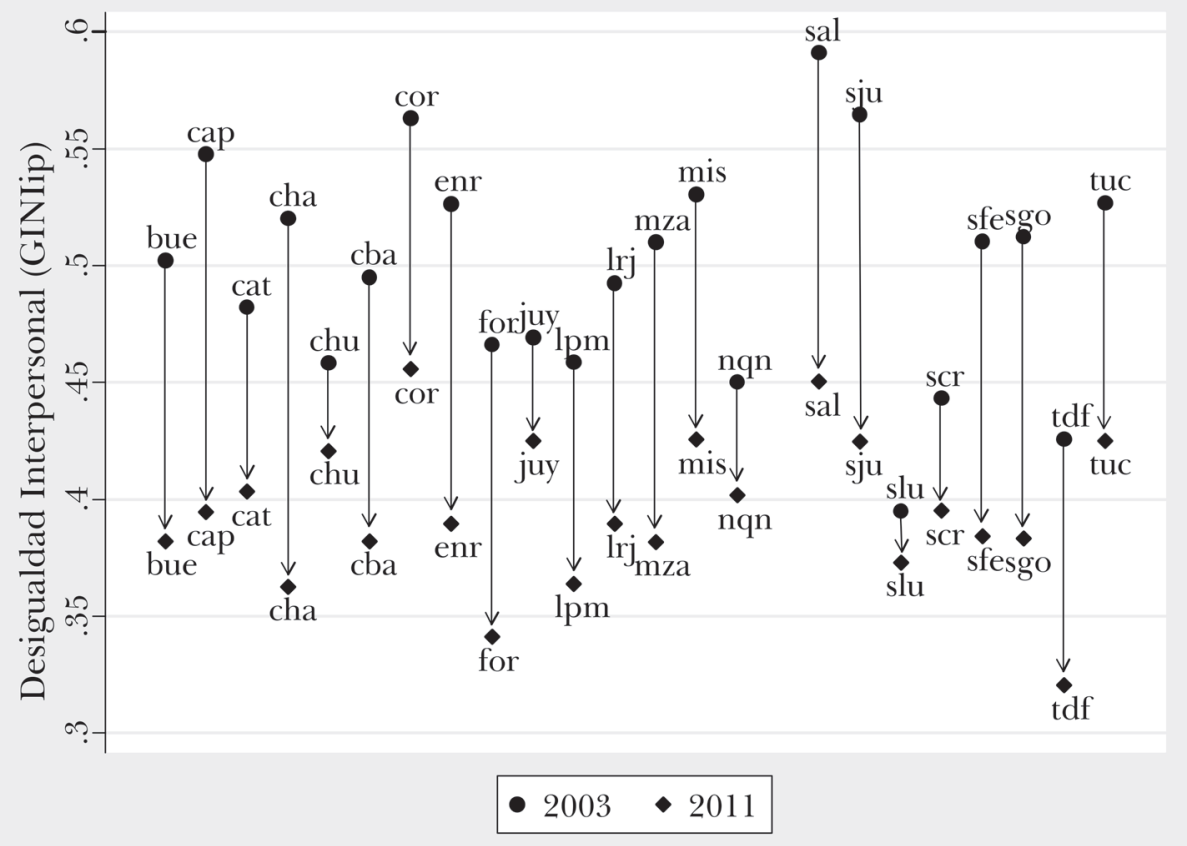

Fuente: elaboración propia en base a datos de EPH de Calvo y Moscovich (2017).

En las figuras anteriores, también llama la atención el contraste entre la gran disminución en la desigualdad en la provincia de Formosa, que pasó de valores relativamente altos $(0,47$ en 2003) a bajos $(0,34$ en 2011) y la provincia de San Luis que redujo su desigualdad de 0,39 a 0,37 en el mismo período. Formosa, aunque empezó siendo más desigual, terminó siendo más igualitaria que San Luis.

También cabe resaltar la variación dentro de provincias relativamente igualitarias y estructuralmente similares. Tierra del Fuego, siendo relativamente igualitaria durante toda la serie, redujo su desigualdad en 10 puntos. Esta es la provincia menos desigual del país para el último año de la serie. Por el contrario, Chubut, Neuquén y Santa Cruz, teniendo niveles de desigualdad parecidos a Tierra del Fuego al comienzo de la serie, redujeron la misma en sólo la mitad (entre 4 y 5 puntos) (ver Tabla 1 y Figuras 2 y 3 ). 
Revista SAAP $\cdot$ Vol. 13, № 1

Tabla 1

Variación de la Desigualdad

\begin{tabular}{|l|l|l|}
\hline & $\begin{array}{l}\text { Alta variación de la } \\
\text { desigualdad }\end{array}$ & $\begin{array}{l}\text { Baja variación de la } \\
\text { desigualdad }\end{array}$ \\
\hline Alta desigualdad $^{6}$ & Salta & Corrientes \\
\hline Baja desigualdad $^{\text {Tierra del Fuego }}$ & San Luis; Santa Cruz \\
\hline
\end{tabular}

Fuente: elaboración propia.

Habiendo analizado los casos y su variación a lo largo del tiempo, nos ocuparemos ahora de establecer algunas precisiones conceptuales, de identificar algunos ejes de discusión teórica y de presentar algunos debates en la literatura sobre el tema.

\section{La desigualdad en el nivel subnacional: aspectos analítico-conceptuales y antecedentes}

¿Qué hace aumentar o disminuir la desigualdad interpersonal? ¿Cómo pueden explicarse las diferencias en la desigualdad entre distintas unidades político-administrativas? Estas son preguntas que han recibido muchísima atención en los últimos años en el nivel nacional. Los desarrollos en este terreno son vastos, tanto en términos teóricos como empíricos. Sin embargo, los trabajos que las han abordado en el nivel subnacional son mucho más escasos y recientes. Mientras en el nivel nacional lo que interesa es la evolución de la desigualdad medida nacionalmente y las diferencias entre los distintos países, cuando se considera el nivel subnacional lo que interesa es fundamentalmente su evolución en los diferentes distritos y las diferencias entre ellos dentro de un mismo país (lo que por supuesto no inhibe la comparación a este respecto entre países diferentes).

Las desigualdades en el plano subnacional pueden ser estudiadas a través de dos grandes vías de abordaje o perspectivas de análisis. Por un lado, se pueden explorar las desigualdades interjurisdiccionales o interregionales, esto es, las diferencias que los distritos dentro de un mismo país (principalmente provincias o estados) muestran respecto de una serie de indicadores como por ejemplo el PBI per cápita, el ingreso personal medio, el índice de desarrollo humano, o el acceso a servicios sociales básicos. Los trabajos que se ubican en 
esta línea de indagación en América Latina se han ocupado, principalmente, de establecer la incidencia que sobre aquellas diferencias tienen las transferencias de los gobiernos centrales y el gasto de diferente tipo que estos gobiernos realizan hacia y en los diferentes distritos subnacionales (ver por ejemplo: González, 2014; Díaz Cayeros 2004 y Rodríguez Silveira 2009). Más recientemente algunos trabajos se han focalizado en identificar algunos factores políticos que inciden sobre las políticas de distribución de recursos nacionales entre los distritos subnacionales (González, 2016 a; González, 2016 b). Si bien se trata de una perspectiva de gran importancia para abordar las cuestiones vinculadas con la desigualdad en los sistemas federales, no es ésta la mirada en la que estamos interesados prioritariamente aquí 6 .

Nuestro interés se centra en las desigualdades intrajurisdiccionales o intrarregionales en el nivel subnacional. Si en las diferentes federaciones americanas (incluyendo Estados Unidos) se observa una amplia dispersión de las desigualdades entre las unidades subnacionales, la pregunta relevante que surge es cuáles son los factores de los cuales depende esta variación.

Por cierto, estos dos aspectos de la desigualdad subnacional —la intery la intrajurisdiccional - están vinculadas. Por un lado, de ambas depende el nivel de desigualdad global de la federación: si la desigualdad interna en todas las unidades es muy similar (digamos bajo), pero la desigualdad interregional es muy alta (es decir, los ingresos medios en cada región son muy diferentes), el nivel total de desigualdad estará (eventualmente muy) por encima de la media de las distintas unidades. Lo mismo sucedería si la desigualdad interregional es muy baja, pero la desigualdad interna de un número importante de jurisdicciones es muy alta. En otras palabras, es imposible reducir sustancialmente la desigualdad total sin reducir simultáneamente tanto la desigualdad inter como intrarregional.

Por otro lado, ambas desigualdades se vinculan con los intereses redistributivos de los diferentes grupos de ingresos en las distintas unidades subnacionales y las correspondientes políticas de redistribución que tales grupos impulsan o defienden (Beramendi, Rogers y Díaz-Cayeros, 2017). Así, por ejemplo, como señalan estos autores, tanto los sectores de altos como de bajos ingresos de una región rica y relativamente igualitaria no apoyarían una política nacional de redistribución tanto intra como

6 En el caso de la Argentina, es ampliamente reconocido que las desigualdades interdistritales en diferentes dimensiones son muy amplias y que se han sostenido a lo largo del tiempo (Kessler 2014: 213-214). Es evidente la importancia que estas desigualdades tienen cuando se piensa en políticas orientadas a reducir las desigualdades en un determinado país. Por más que se adoptaran políticas nacionales intensas de redistribución a nivel personal, la reducción de las desigualdades interpersonales encontraría un umbral relativamente alto si las desigualdades interdistritales se mantuvieran relativamente elevadas. 
interregional de la que obtendrían poco o ningún beneficio y a la que tendría que aportar buena parte de sus recursos ${ }^{7}$. Más allá de estas conexiones entre ambos tipos de desigualdades, la distinción analítica entre ellas es clara, lo que habilita a tratarlas separadamente como objeto de investigación.

\section{Tipos de factores que inciden sobre las desigualdades subnacionales}

Respecto a los factores que pueden explicar las diferencias en las desigualdades intradistritales en un país, podemos pensar, tomando como referencia la literatura que se ha ocupado de la desigualdad a nivel nacional, en una distinción entre factores socioeconómicos y demográficos, de política pública y fiscales, y político-institucionales. En este caso los factores son clasificados según el «ámbito» sociopolítico al que pertenecen. A su vez, y dado que cuando nos ocupamos del nivel subnacional, es razonable pensar en influencias recíprocas entre este nivel y el nacional, podemos establecer una segunda distinción según aquellos factores operen solamente en el nivel subnacional (factores «internos»), o bien se trate de factores nacionales que operan diferencialmente en los diferentes distritos subnacionales (factores «externos») (ver al respecto Giraudy et al., en prensa). En este caso los factores son clasificados según el nivel en el que tienen su «origen». De este modo tendríamos seis tipos de factores que teóricamente podrían afectar la desigualdad en el nivel subnacional, tal como muestra la Tabla 2.

Los factores socioeconómicos y demográficos, político-institucionales, fiscales y de política pública están, sobre todo estos dos últimos, fuertemente relacionados ${ }^{8}$. No obstante, a los fines analíticos, en lo que sigue de este

7 En las federaciones, si bien las unidades políticas subnacionales que tienen capacidad para interactuar con el nivel nacional son los estados o provincias, también pueden incorporarse en este análisis a los gobiernos locales en aquellos países en los cuales tienen ciertas prerrogativas para asignar o reconducir el gasto público estadual o federal. Un estudio de este tipo es el de Arretche (2009) sobre Brasil, que muestra el efecto relevante que la existencia o no de regulaciones nacionales y estaduales tienen sobre la asignación del gasto por parte de los gobiernos locales y, con ello, sobre el impacto que este tiene en términos de la desigualdad a nivel local.

8 En general, puede asumirse que las políticas fiscales y la política pública actúan como variables intermedias a través de las cuales se canaliza la incidencia de los factores político-institucionales sobre las desigualdades. En efecto, parece razonable pensar que buena parte de los efectos de variables como "ideología del gobierno" o "democracia" afectan a la desigualdad a través del efecto que tienen sobre el gasto y el contenido de las políticas públicas. Por su parte, los factores socio-económicos y demográficos, al tiempo que son influidos por las políticas públicas, son también la base "estructural" que configura los "límites" en los que se desarrollan los procesos políticos y las acciones estatales resultantes. Sobre esta relación entre aspectos estructurales y los propiamente políticos ver Oszlak y O’Donnell (1976) y Acuña (2013) 
apartado realizaremos algunas breves consideraciones conceptuales y metodológicas sobre los diferentes tipos de factores prescindiendo de las relaciones entre ellos. Por cuestiones de viabilidad analítica y metodológica, aquí solamente nos focalizaremos en los factores subnacionales (internos) y especialmente los fiscales y político-institucionales (que englobaremos bajo el término factores políticos) ${ }^{9}$.

\section{Tabla 2}

Tipos de factores explicativos de las desigualdades subnacionales

\begin{tabular}{|l|l|l|}
\hline $\begin{array}{l}\text { Nivel } \\
\text { Ámbito }\end{array}$ & $\begin{array}{l}\text { Nacionales } \\
\text { (“externos") }\end{array}$ & $\begin{array}{l}\text { Sub-Nacionales } \\
\text { (“internos") }\end{array}$ \\
\hline $\begin{array}{l}\text { Socio-económicos } \\
\text { y demográficos }\end{array}$ & $\begin{array}{l}\text { Rasgos nacionales } \\
\text { agregados de la población, } \\
\text { la economía y el mercado } \\
\text { de trabajo }\end{array}$ & $\begin{array}{l}\text { Distribución demográfica } \\
\text { diferencial y de la oferta y } \\
\text { demanda laboral entre distritos } \\
\text { subnacionales }\end{array}$ \\
\hline $\begin{array}{l}\text { Fiscales y de } \\
\text { Política Pública }\end{array}$ & $\begin{array}{l}\text { Estructura fiscal, políticas } \\
\text { públicas y transferencias } \\
\text { nacionales en y hacia } \\
\text { distritos subnacionales }\end{array}$ & $\begin{array}{l}\text { Gasto y políticas públicas de los } \\
\text { gobiernos subnacionales en sus } \\
\text { distritos }\end{array}$ \\
\hline $\begin{array}{l}\text { Político- } \\
\text { Institucionales }\end{array}$ & $\begin{array}{l}\text { Marco institucional y } \\
\text { dinámica política } \\
\text { nacionales }\end{array}$ & $\begin{array}{l}\text { Marco institucional y dinámica } \\
\text { política subnacionales }\end{array}$ \\
\hline
\end{tabular}

Fuente: elaboración propia.

$9 \quad$ Por cierto, el abordaje de las desigualdades subnacionales exige la consideración tanto de los factores internos como externos. Sin embargo, estos últimos plantean desafíos teóricos y metodológicos específicos que no estamos en condiciones de abordar de modo simultáneo con los que plantean los factores internos, los cuales elegimos priorizar en este trabajo. Sólo diremos sobre los factores que operan en el nivel nacional que la literatura sobre ellos es prácticamente inexistente. Hasta dónde sabemos, para América Latina sólo podemos incluir como un antecedente al respecto el trabajo de Cont y Porto (2014) quienes establecen, para el caso argentino, el efecto progresivo del gasto nacional en las provincias (lo que implica que este gasto opera como un factor explicativo de las diferencias en los niveles de desigualdad en las diferentes jurisdicciones provinciales). Respecto a considerar prioritariamente los ámbitos fiscal y político-institucionales, además de razones de formación disciplinar, hay razones sustantivas: los factores socioeconómicos tienen una incidencia específica sobre la desigualdad y una dinámica propias, pero en la medida en que, al menos parcialmente, puedan ser modificados o direccionados, lo serán en buena parte (si bien no únicamente) como resultado de políticas públicas y de los procesos políticos que subyacen a las diferentes acciones del Estado. 


\section{Los factores políticos subnacionales}

Respecto a estos factores creemos pertinente hacer una referencia de orden teórico-metodológico. En primer lugar, podemos suponer que existe una homología estructural, respecto a la desigualdad y sus variables explicativas, entre el nivel nacional y el subnacional. Esto es, la desigualdad en ambos niveles tiene una dinámica similar y las variables explicativas son básicamente las mismas, sólo que operando en niveles diferentes. De este modo, las diferencias subnacionales en la desigualdad se explican básicamente por una distribución diferencial, entre unidades subnacionales, de los valores de ciertos factores explicativos que operan en el nivel nacional, del mismo modo que las diferencias nacionales resultan de una distribución diferencial entre países de los valores de los mismos factores. Dado que los desarrollos teóricos y sus contrastaciones empíricas se han producido hasta ahora casi exclusivamente en el nivel nacional, en esta variante de factores internos lo que tenemos en realidad es un traslado teórico y metodológico desde el nivel nacional hacia el nivel subnacional. Esta estrategia de «traslado», además de permitir aprovechar desarrollos teóricos y metodológicos previos, es razonable desde un punto de vista analítico. En efecto, en principio se puede asumir que lo que es válido en el nivel nacional opera de un modo similar, al menos parcialmente, también en el nivel subnacional. Como veremos, los muy escasos trabajos que abordan los factores políticos que explican las desigualdades subnacionales lo hacen a partir de esta estrategia de traslado (si bien usando para este traslado teorías diferentes del «menú» teórico hoy disponible en el nivel nacional).

Sin embargo, en segundo lugar, esta estrategia presenta problemas potencialmente serios. Como señala Rodrigues-Silveira (2013), algunos autores han remarcado los peligros de utilizar mecánicamente conceptos pensados para una escala de análisis (por ejemplo, nacional) en otra escala (por ejemplo, local o provincial). Esto se debe a que la definición y la referencia empírica de los conceptos en una escala puede no ser adecuada u operativa en otra, pudiendo dar a lugar a un «estiramiento conceptual», según lo define Sartori (1970, citado en Rodrígues-Silveira, 2013: 12-13). Además, las relaciones causales entre variables pueden cambiar drásticamente, de modo que una relación entre ellas en un nivel o escala, desaparezca o se invierta en otra (2013: 14).

Siguiendo esta misma línea de razonamiento, agregamos una tercera complicación: podemos pensar que hay ciertas variables explicativas que están presentes solamente en el nivel subnacional, en tanto es aceptable suponer que la dinámica política en este nivel no es idéntica a la dinámica política en el nivel nacional, particularmente en sistemas federales con una 
marcada autonomía político-electoral y administrativa para sus niveles subnacionales. Esto es, la estrategia del traslado corre el riesgo de omitir importantes variables explicativas.

En definitiva, las diferencias en las desigualdades intradistritales no pueden explicarse sólo por la distribución diferencial de factores que son válidos en el nivel nacional, lo que hace necesario una reflexión teórica y metodológica sobre los factores específicamente provinciales (y locales) que afectan la desigualdad dentro de cada distrito subnacional, que complemente, adecúe y eventualmente reformule los marcos analíticos derivados de reflexiones sobre el nivel nacional. Hasta dónde sabemos esta es una línea de indagación sobre las desigualdades subnacionales que aún no ha sido explorada.

Dado que la estrategia del traslado es la que ha sido utilizada por la literatura hasta ahora existente sobre la relación entre factores políticos y desigualdad en el nivel subnacional, en lo que sigue de este apartado presentaremos sumariamente las teorías más importantes sobre esta cuestión en el nivel nacional para ocuparnos luego brevemente del uso que aquella literatura hizo de las mismas.

\section{Desigualdad y política: teorías «nacionales» y su aplicación subnacional}

La enorme cantidad de trabajos que, en diferentes contextos, se han ocupado de la relación entre factores político-institucionales y la desigualdad en el nivel nacional, son tributarios en su gran mayoría de una cantidad acotada de teorías que definen las variables relevantes a tener en cuenta para explicar la evolución de los niveles de desigualdad en un mismo país y/ o dar cuenta de las diferencias entre diferentes países. Estos trabajos y desarrollos teóricos pueden agruparse (sin pretensión de exhaustividad) en tres grandes grupos de teorías nacionales «disponibles» para el traslado al nivel subnacional: la teoría del votante medio (TVM), la teoría de los recursos de poder (TRP) y las teorías político-institucionales (TPI) ${ }^{10}$.

De manera muy sintética, la TVM sostiene que cuando mayor sea la desigualdad «de mercado» en una democracia y, por ende, mayor el ratio ingreso del votante medio/ingreso medio, habrá una presión redistributiva que llevará al descenso (progresivo) de la desigualdad «después» de la intervención del Estado por medio de transferencias e impuestos (Meltzer y Richard 1981).

10 En lo que sigue haremos referencia al núcleo argumental de estas perspectivas teóricas, sin adentrarnos en las contrastaciones empíricas (no siempre favorables) de las que han sido objeto ni en los ajustes y argumentos complementarios que se han desarrollado para dar cuenta de algunas "anomalías" que surgieron de diferentes trabajos empíricos. 
La TRP, por su parte, propone que la desigualdad será menor cuanto mayor y más potente sea la organización sindical y política de la clase obrera, lo que le permitirá reducir la desigualdad de mercado y a la vez presionar por políticas redistributivas (Korpi 1983, Stephens 1979, Esping-Andersen 1990). Esto es, sindicatos poderosos y partidos laboristas o socialdemócratas en el gobierno estarán asociados con menores niveles de desigualdad, tanto antes como después de la intervención estatal a través de impuestos y transferencias (Bradley et. al. 2003) ${ }^{11}$.

Finalmente, las TPI señalan la importancia de las reglas electorales. Los sistemas proporcionales tienen dos efectos. Por un lado, abren la representación a los sectores menos favorecidos económicamente (Birchfield y Crepaz 1998). Por el otro, incentivan a los sectores medios a aliarse con aquellos sectores de bajos ingresos (ya que disminuyen, para las clases medias, el riesgo, presente en un sistema mayoritario, de que un gobierno de izquierda tenga los recursos políticos que le permitirían avanzar sobre sus propios ingresos) (Iversen y Soskice, 2006; Austen-Smith, 2000; Schneider y Soskice 2009; Ardanaz and Scartascini, 2013). Un corolario de esta perspectiva es que los sistemas políticos más competitivos (en los cuales la diferencia en porcentajes de votos entre el primero y el segundo sea relativamente pequeña), más propio de los sistemas proporcionales, estarán asociados con relativamente menores niveles de desigualdad ${ }^{12}$.

Como señalamos más arriba, los muy escasos trabajos (un total de tres) que se ocupan de los factores políticos y su incidencia sobre la desigualdad subnacional en países de América han usado la estrategia del traslado teórico desde el nivel nacional. Ninguno de ellos utiliza la TVM (ni la TDS), los tres

11 Calvo y Moscovich (2007) proponen lo que puede considerarse una variante, hasta cierto punto crítica, de la TRP. Según estos autores, en coincidencia con la TRP, la sola existencia de niveles altos de desigualdad no dispara automáticamente mecanismos político-electorales para su reducción, como sostiene la TVM. La incidencia de niveles altos de desigualdad sobre la implementación de políticas redistributivas dependerá de la "visibilidad" que aquellos niveles adquieren en la arena pública, y esta visibilidad depende en buena medida del grado de movilización y protesta de los sindicatos y los sectores más perjudicados por la estructura de distribución de ingresos. Esto es, los procesos redistributivos y los niveles de desigualdad resultantes dependen de la protesta de manera crítica, y no sólo de la fortaleza electoral y sindical de las clases subordinadas.

12 A estos tres grupos de teorías podríamos agregar un cuarto, más reciente y menos difundido, que pone el acento en los aspectos subjetivos que definen el modo en que las clases medias y altas ven y perciben a los sectores menos favorecidos. Lo que podríamos llamar teoría de la distancia social (TDS) sostiene que la formación de coaliciones redistributivas dependerá de la distancia social relativa que separa a las clases medias de las bajas (Lupu y Pontusson, 2011) o a las clases altas de las bajas (Blofield, 2011). Cuando estas distancias sociales son relativamente cortas, se implementarán políticas redistributivas que reducirán la desigualdad. A la inversa, cuando estas distancias son amplias, se mantendrá el statu quo distributivo e incluso pueden darse procesos de redistribución regresiva. 
recurren a la TRP (si bien de diferente modo y con diferentes resultados según las expectativas de la teoría) y uno, además utiliza una variante de las TPI. Como veremos, no se trata de traslados mecánicos de las teorías nacionales, ya que en los tres casos hay aportes analíticos y empíricos que incorporan, al menos parcialmente, la especificidad de la escala subnacional, tanto en su propia particularidad como en su relación con la escala nacional. Aun así, como remarcaremos también más adelante, la «perspectiva nacional» es preponderante y la especificidad subnacional queda en un muy segundo plano.

Calvo y Moscovich (2017) se ocupan del caso argentino y adoptan la versión de la «protesta» de la TRP ${ }^{13}$. Los autores encuentran que cuanto mayor es el nivel de protesta en el nivel provincial, mayor es el efecto redistributivo progresivo de las transferencias condicionadas de ingreso (y, consiguientemente, menor es la desigualdad a igualdad de otros factores). Este efecto es mayor cuanto mayor es el nivel «previo» de desigualdad. El aporte específico al análisis subnacional de este trabajo es que muestra que los factores externos (en este caso, una política pública del gobierno nacional) no tienen un efecto directo sobre las desigualdades en el nivel nacional, sino que el mismo está mediatizado por una variable (en este caso «protesta») que opera diferencialmente en los distintos escenarios provinciales.

Por su parte, Kelly y Witko (2012), asumen explícitamente la versión original de la TRP «nacional» en su abordaje de las diferencias en la desigualdad de ingresos entre los estados de Estados Unidos. Consecuentemente, utilizan como variables explicativas de los niveles de desigualdad en los estados, por un lado, la «densidad sindical» en estas unidades subnacionales (como indicador de la fortaleza de la clase obrera en el ámbito del mercado) y, por el otro, la «orientación ideológica de izquierda» de los gobiernos estatales (como un indicador de los recursos de poder de la clase obrera en el ámbito político). Encuentran que ambas variables tienen un efecto negativo y estadísticamente significativo sobre la desigualdad (tanto previa como posterior a impuestos y transferencias), si bien la variable política tiene significación sólo en el período de preminencia de los congresos y/ o gobiernos federales que expresan los intereses de las clases de ingresos más altos. Esto se debe a la orientación de los gobiernos republicanos a descentralizar competencias desde el nivel federal hacia los estados, y al esfuerzo de los demócratas de darles a estos, más autoridad para habilitar

13 Para Calvo y Moscovich (2017) esta perspectiva no queda incluida en la TRP, sino que puede considerarse crítica "externa" a la misma (y a la TVM). No se trata ciertamente de una cuestión sustancial, pero desde nuestro punto de vista la "visualización" de la desigualdad a través de la protesta afecta el balance de poder entre los grupos de ingresos que tienen una relación esencialmente conflictiva sobre la redistribución, lo cual hace compatible esta perspectiva con el marco general de la TRP, cuyo alcance contribuye a ampliar. 
en ellos la opción de políticas más equitativas que equilibren las políticas más regresivas del gobierno federal cuando este está en manos republicanas. Se trata, entonces, de una interacción entre las variables políticas nacionales y subnacionales por la cual, cuando hay supremacía demócrata a nivel nacional, dada la orientación redistributiva del gobierno federal, se reduce la incidencia de las variables estatales sobre la desigualdad subnacional. A la inversa, cuando se da la supremacía republicana en la esfera federal, se hace más importante el efecto de las variables políticas estatales, en tanto los gobiernos subnacionales hacen un mayor esfuerzo, bajo ciertas condiciones políticas internas y en un contexto de mayores recursos y competencias administrativas, para compensar la redistribución (más) regresiva impulsada por el gobierno federal (Kelly y Witko 2012: 418). Es en esta interacción que el trabajo transciende una mera «aplicación» de la TRP a la escala subnacional, para establecer la relevancia específica de este nivel, no contemplado originalmente por la teoría.

Finalmente, Sátyro (2013) aborda la explicación de las diferencias en las desigualdades de ingreso entre los estados de Brasil. La autora examina la incidencia de dos variables políticas: la ubicación en el espectro ideológico de los gobiernos estaduales (derivada de la TRP) y la competitividad electoral (derivada de la TPI en su variante del consenso) ${ }^{14}$. Su análisis empírico muestra que la variable ideológica, si bien significativa, tiene un sentido opuesto al postulado por la teoría, ya que los partidos de centro y centro derecha producen mayores reducciones de desigualdad que el partido de izquierda de referencia (el Partido de los Trabajadores). Por su parte, la variable electoral si bien tiene un efecto bajo, el mismo es significativo y tiene el sentido predicho por la teoría: a mayor concentración de votos en el candidato que obtiene el primer lugar (menor competitividad), mayor desigualdad. A su vez, este efecto se da a través de la reducción de la informalidad en el mercado de trabajo.

En primer lugar, este trabajo muestra que el efecto de los factores políticos sobre la desigualdad en el nivel subnacional se da a través de cambios en el mercado de trabajo (algo que también muestran Kelly y Witko, 2012, para el caso norteamericano cuando constatan la incidencia del «salario mínimo» sobre la desigualdad). Esto es muy relevante porque hasta ahora se consideraba que los niveles subnacionales tenían poca relevancia respecto de las características del mercado laboral. En segundo lugar, ilustra empíricamente los peligros, señalados más arriba, de asumir linealmente los postulados teóricos y los hallazgos de teorías válidas en el nivel nacional (en este

14 Sátyro (2013) propone que estas variables políticas inciden sobre la desigualdad a través de dos variables que afectan la inserción de los sectores de menores recursos en el mercado laboral: nivel de analfabetismo y grado de informalidad laboral. 
caso la TRP) cuando se dan cambios de escala hacia el nivel subnacional.

Creemos pertinente concluir la revisión de estos trabajos señalando tres cuestiones que nos parecen relevantes. En primer lugar, todos ellos usan como variables de control algunas variables socioeconómicas tales como población y necesidades básicas insatisfechas (Calvo y Moscovich 2017), desempleo, porcentaje de población de edad avanzada y población no blanca, producción manufacturera (Kelly y Witko, 2012), y PBI per capita e infraestructura sanitaria (Sátyro, 2013). Todas ellas son idénticas o muy similares a las utilizadas por los estudios que se ubican nivel nacional (ver, por ejemplo, Huber y Stephens, 2012, para América Latina, y Bradley, et. al., 2003, para los países desarrollados). Esto es, respecto de las variables socioeconómicas, estos trabajos usan también la estrategia del traslado del nivel nacional al subnacional.

En segundo lugar, ninguno de los trabajos incorpora en sus análisis, factores fiscales y de gasto público, a pesar de que estos factores (particularmente el gasto social) han sido muy usados en estudios nacionales (por ejemplo, Huber y Stephens, 2012, para América Latina), con lo cual podemos decir que hay cierta inconsistencia en la aplicación de la estrategia de traslado elegida.

Finalmente, como señaláramos más arriba, estos trabajos hacen una importante contribución al desarrollo de una temática muy poco explorada, en tanto examinan la validez de teorías «nacionales» sobre la relación entre política y desigualdad en el nivel subnacional. Al mismo tiempo, trascendiendo la aplicación mecánica de estas teorías, establecen algunas de las contribuciones específicamente subnacionales a esta relación al aportar evidencia de la interacción entre factores nacionales y subnacionales, de una parte, y al precisar el modo específico en que algunas variables políticas se relacionan con la desigualdad en la escala subnacional, de la otra.

Sin embargo, en ninguno de ellos se explora la posibilidad de que variables políticas específicas de la escala subnacional (que no estarán presentes en el nivel nacional) incidan sobre la desigualdad. En términos teóricos y metodológicos esta posibilidad es muy relevante, ya que en ella se expresaría con claridad en qué medida el espacio político subnacional tiene particularidades tales en su vínculo con la desigualdad que lo constituyen en un ámbito estructuralmente diferenciado, a este respecto, del espacio político nacional. En este sentido, nos parece pertinente señalar que los modelos estadísticos presentados en estos trabajos explican una proporción relativamente baja de la variación de la desigualdad o los efectos redistributivos del gasto social, lo que implica la existencia de importantes variables explicativas que han sido omitidas.

Desde nuestro punto de vista, esto expresa que la especificidad de las dinámicas políticas subnacionales y el modo a través del cual inciden sobre las desigualdades en esta escala son cuestiones de gran relevancia, y que su 
abordaje teórico y empírico, hoy aún pendiente, forman parte central de una agenda de investigación sobre este tema.

Con esto en mente, en el apartado siguiente exploramos, a partir de los datos disponibles hasta el momento, la relevancia empírica de algunas variables internas y externas para explicar las diferencias en los niveles de desigualdad entre las provincias argentinas.

\section{La explicación de la desigualdad en las provincias argentinas: algunos resultados significativos}

En este apartado examinamos la relación de algunas variables que han sido destacadas por la literatura (tanto «nacional» como «subnacional») con la desigualdad de ingreso, más otras variables no estudiadas hasta ahora pero que, desde nuestra perspectiva, pueden permitir algún avance en el establecimiento de la especificidad del nivel subnacional. Esta exploración está condicionada por los datos disponibles hasta el momento, pero creemos que aún con esta limitación, es posible establecer algunos primeros resultados importantes para la construcción de una agenda de investigación. En primer lugar, examinamos variables socioeconómicas, luego variables fiscales y, finalmente, variables políticas, señalando, en cada caso, si se trata de variables externas o internas. La Tabla 3 muestra los resultados de correlación entre la desigualdad y algunas variables socioeconómicas, políticas y fiscales.

\section{Tabla 3}

Desigualdad (coeficiente de Gini) y Coeficientes de Correlación con variables seleccionadas

\begin{tabular}{|c|c|c|}
\hline \multicolumn{2}{|r|}{ Variables } & \multirow{2}{*}{$\begin{array}{c}\text { Correlación } \\
0,664^{* * *}\end{array}$} \\
\hline Variables & Desempleo & \\
\hline $\begin{array}{l}\text { Soc10- } \\
\text { económicas }\end{array}$ & Empleo Agrícola & $0,435^{* * *}$ \\
\hline & Pobreza & $0,429 * * *$ \\
\hline \multirow{3}{*}{$\begin{array}{l}\text { Variables } \\
\text { Fiscales }\end{array}$} & Gasto público social per cápita & $-0,658^{* * *}$ \\
\hline & Transferencias nacionales & $-0,602 * * *$ \\
\hline & Empleo público & $-0,445^{* * *}$ \\
\hline \multirow{3}{*}{$\begin{array}{l}\text { Variables } \\
\text { Políticas }\end{array}$} & Porcentaje de votos al gobernador & $-0,298 * * *$ \\
\hline & Diferencia de votos entre el partido del gobernador y el segundo & $-0,324 * * *$ \\
\hline & Años del partido del gobernador en el poder & $-0,296^{* * *}$ \\
\hline
\end{tabular}

Fuente: elaboración propia. 
Dentro del primer grupo de variables, los coeficientes indican que, como esperaría una parte de la literatura, a medida que aumenta el desempleo y la pobreza tiende a aumentar la desigualdad en las provincias (ver Gráfico 4). Los coeficientes son relativamente robustos y estadísticamente significativos. Encontramos también otras variables socioeconómicas que correlacionan positivamente con desigualdad, como analfabetismo $(0,35)$ y mortalidad infantil $(0,37)^{15}$. Es decir, provincias con problemas socioeconómicos más serios, tienden a tener niveles de desigualdad mayores.

\section{Gráfico 4}

Desigualdad y Desempleo en las Provincias Argentinas (2003-2011)

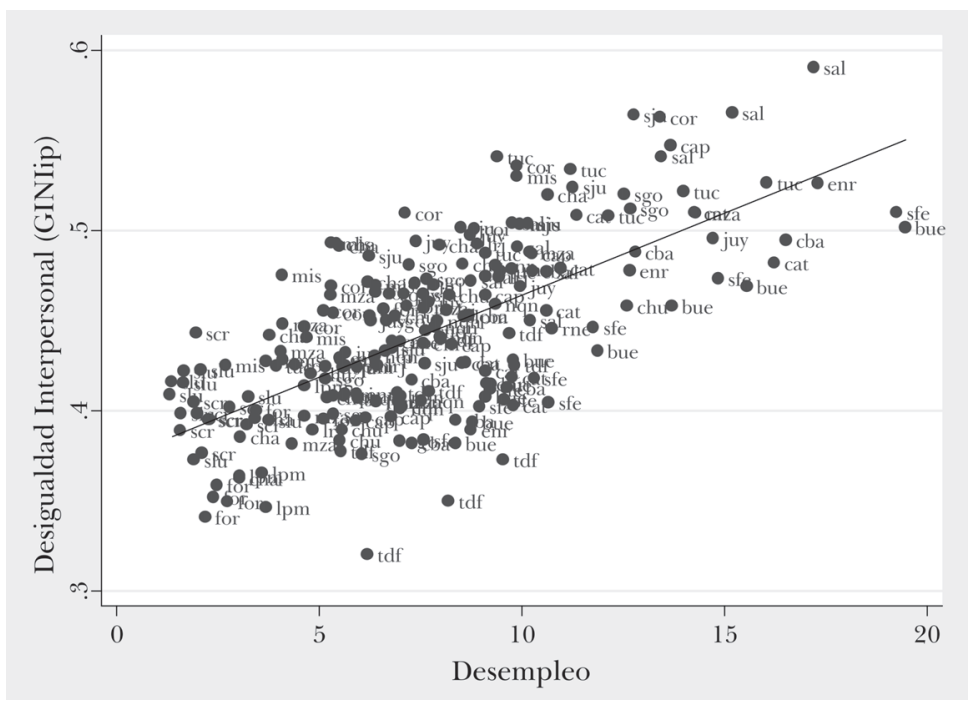

Fuente: elaboración propia.

Los resultados reportan también una relación positiva entre crecimiento de la economía nacional y desigualdad $(0,26)$, a pesar de que una parte de la literatura esperaría lo contrario. Sin embargo, no encontramos relación entre variación del producto bruto geográfico provincial (per cápita) y la desigualdad.

15 Nivel de urbanización correlaciona negativamente (-0,28). Estos coeficientes no fueron reportados en la tabla para ahorrar espacio. 
Además, observamos que hay una relación entre desigualdad y tipo de empleo. En particular, es positiva la relación entre desigualdad y proporción de empleo agrícola sobre empleo total. Llamativamente, no hay relación entre empleo industrial y desigualdad. Pero encontramos una relación negativa y robusta entre cantidad de empleados públicos cada 1.000 habitantes y desigualdad. Esto puede ser así porque el empleo público ha construido una «malla social» que ofrece cierta contención a los sectores de ingresos bajos y medios Estudios futuros podrán indagar en mayor detalle las relaciones entre los tipos de empleo y la desigualdad.

Otra parte de la literatura sugiere que la desigualdad se relaciona con variables fiscales, como déficit nacional y provincial. Contrario a lo que esperaría una parte de la literatura, encontramos una relación positiva y estadísticamente significativa entre déficit (tanto nacional como provincial) y desigualdad $(0,49$ y 0,38): es decir, cuanto mayor es el superávit, más aumenta la desigualdad. Estos resultados podrían indicar que la desigualdad tiende a aumentar en períodos de mayor conservadurismo fiscal, pero estudios en mayor profundidad podrían llegar a precisar mejor la relación entre estas variables.

Finalmente, tal como esperan Huber y Stephens (2012), encontramos un vínculo entre el tipo de gasto provincial y Gini. Los resultados indican que el gasto público social per cápita de las provincias se relaciona negativamente con desigualdad. Esta relación es robusta y significativa (ver Tabla 3 y Gráfico 5).

Además, las provincias que reciben montos de transferencias nacionales per cápita más altos tienden a ser más igualitarias. Las dos provincias que reciben más transferencias nacionales per cápita, Santa Cruz y Tierra del Fuego (recibieron 3.934 y 3.640 dólares per cápita en 2011, respectivamente), tienen algunos de los valores de desigualdad más bajos del país (ver Tabla 3 y Gráfico 6$)^{16}$.

16 Encontramos una relación positiva $(0,38)$ y significativa entre superávit fiscal y desigualdad, pero no tenemos claros argumentos teóricos para vincular causalmente estas dos variables. 


\section{Gráfico 5}

Desigualdad y Gasto Social en las Provincias Argentinas (2003-2011)

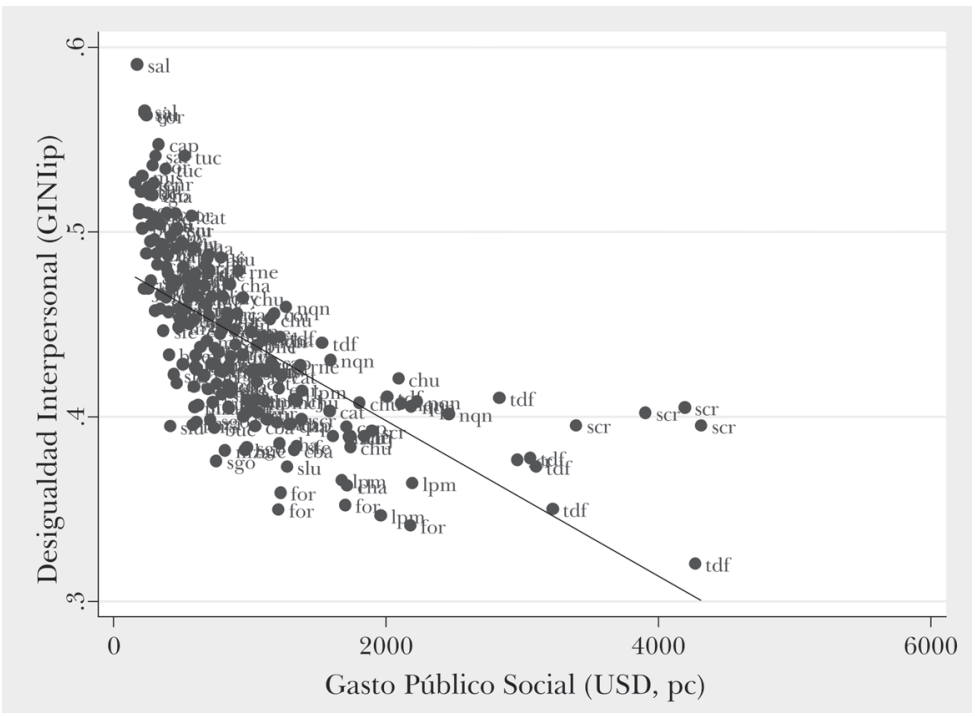

Fuente: elaboración propia.

\section{Gráfico 6}

Desigualdad y Transferencias Nacionales en las Provincias Argentinas (2003-2011)

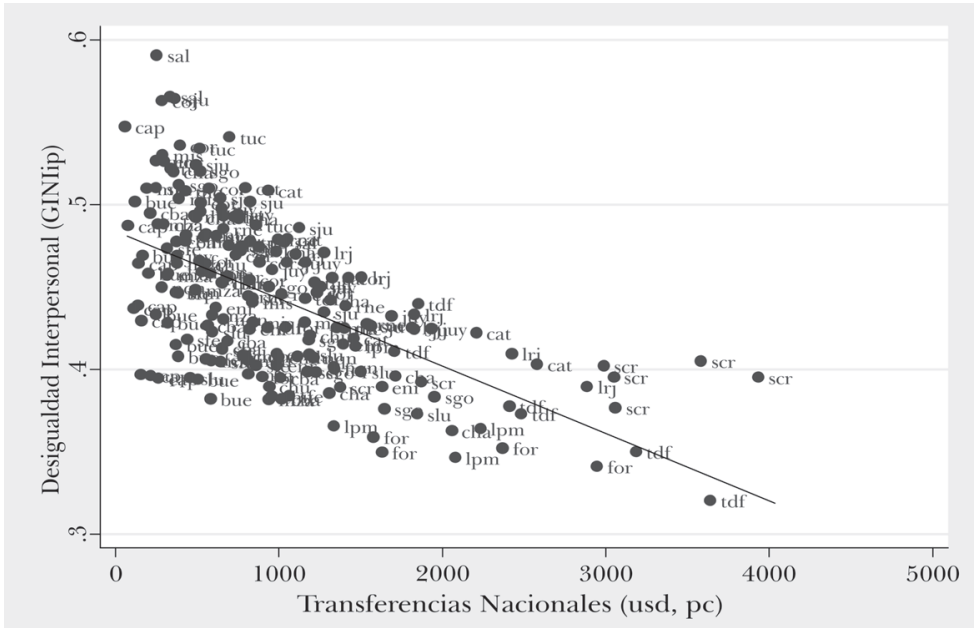

Fuente: elaboración propia. 
En relación al tercer grupo de variables, los factores políticos, varios autores sostienen que reglas mayoritarias tienden a aumentar la desigualdad (Austen-Smith 2000, Persson y Tabellini 2000, Bradley et al. 2003, Iversen y Soskice 2006, Schneider y Soskice 2009, Ardanaz and Scartascini 2013). Corbacho (1998) codificó el «grado de mayoritarismo» de las constituciones provinciales en 1994. Usamos su índice para analizar si existe alguna relación entre éste y la desigualdad provincial. Los resultados reportan que cuanto más mayoritaria es la constitución provincial, mayores son los valores del índice de Gini. La correlación es relativamente robusta (0.27) y estadísticamente significativa.

Observamos también que la desigualdad tiende a ser menor cuanto mayor es el porcentaje de votos del gobernador, cuanto mayor es la diferencia de votos entre el partido del gobernador y el segundo partido, y cuando el partido del gobernador lleva más años en el poder, todo lo cual contradice las expectativas de las TPI. Esto posiblemente esté relacionado con el hecho de que gobernadores más estables en el cargo tienden a hacer más gasto social y menos gasto corriente (como encontramos en trabajos anteriores: González 2017; González y Cáceres 2017) y el gasto social, como vimos anteriormente, correlaciona negativamente con desigualdad (ver Gráfico 7).

\section{Gráfico 7}

Desigualdad y Competencia Electoral en las Provincias Argentinas (2003-2011)

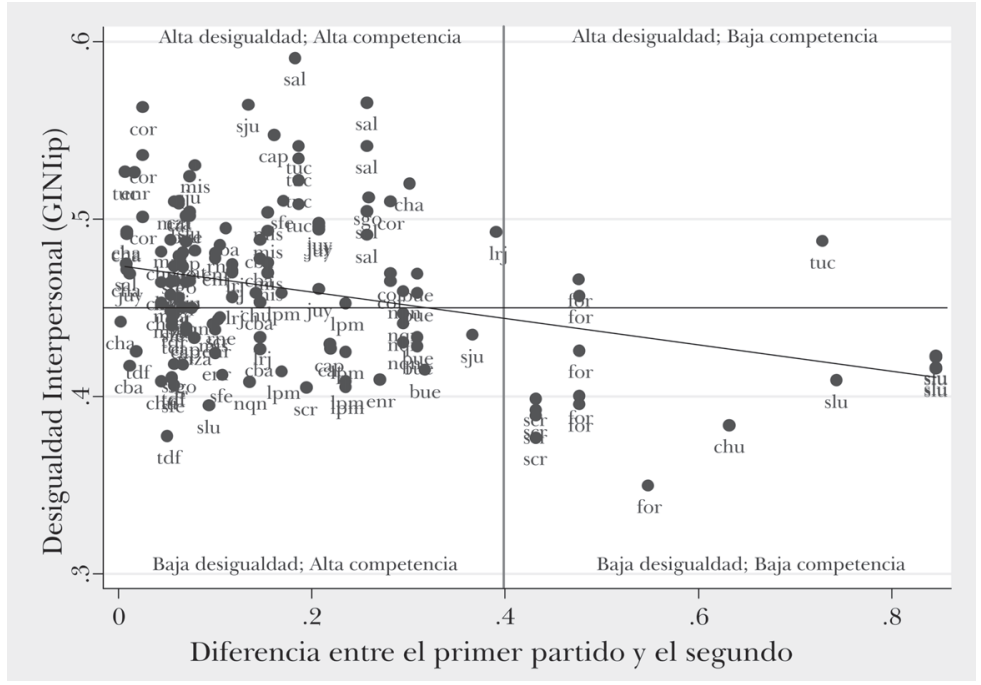

Fuente: elaboración propia. 
Parte de la literatura sostiene que los políticos (en nuestro caso, los gobernadores) con mayor seguridad electoral pueden reducir los costos de transacción de la política y a distribuir bienes públicos a un grupo más amplio de votantes, ya que los beneficios electorales de este tipo de gasto son mayores (como argumentaron Bueno de Mesquita, 2003: 55-56; Chhibber y Nooruddin, 2004: 153-154; Magaloni, Díaz-Cayeros y Estevez, 2007: 188, 201-202 y Persson y Tabellini, 2000). Parte de la literatura en el tema espera este mismo vínculo debido a la menor incertidumbre de las elecciones, la menor necesidad de asegurar votos a través de bienes particularistas y los mayores beneficios electorales del gasto en bienes públicos, como el gasto social (Bueno de Mesquita, 2003; Chhibber y Nooruddin, 2004; Magaloni; Díaz-Cayeros; Estevez, 2007).

En línea con la falta de evidencia de que a nivel provincial operan en nuestro país los supuestos predominantes en la TPI vinculados a los márgenes electorales, no encontramos relación entre fragmentación partidaria (medida con el número efectivo de partidos en votos o bancas) (datos de Varetto, 2014) y desigualdad, a pesar de que una parte de la literatura considera que esta variable incide sobre indicadores sociales. Entre ellos, Chhibber y Nooruddin (2004: 153-154) y Persson y Tabellini (2000) sostienen que los sistemas bipartidistas tienden a generar más gasto en bienes públicos que los sistemas multipartidistas. Esto es así porque, en un sistema bipartidista, los gobiernos enfrentan menos presiones para concentrar sus gastos y proporcionar bienes privados a grupos específicos de votantes para obtener su apoyo.

Finalmente, en línea con lo postulado por la TRP, analizamos si el tipo de partido en el gobierno provincial y su ideología están relacionados con la desigualdad, tal como hicieron Kelly y Witko (2012) en los Estados Unidos y Sátyro (2013) en Brasil. Reconocemos lo complejo del ejercicio de identificación de ideología partidaria en algunas provincias argentinas, fundamentalmente en el caso del Partido Justicialista (PJ). En algunos casos, la definición de su condición de izquierda o derecha puede llegar a depender, en gran medida, del tipo de política pública que hace el partido. Siendo conscientes de este desafío, clasificamos los partidos en el gobierno provincial en seis tipos: Partido Justicialista (PJ), Unión Cívica Radical (UCR), Frente para la Victoria (FPV), Radicales K, Partido Socialista o partidos provinciales (MPN, PACh, PAL, entre otros) ${ }^{17}$.

17 Incluimos al PRO como partido provincial porque solamente tenemos datos hasta el año 2011. En ese año, el PRO gobernaba solamente la Capital Federal y no tenía presencia territorial en otro distrito ni legisladores nacionales por otra provincia (en 2011, el PS tenía legisladores nacionales de tres provincias diferentes y legisladores provinciales en dos). 
No encontramos grandes variaciones en la media de la desigualdad en provincias gobernadas por los dos grandes partidos tradicionales (PJ y UCR). En los dos casos, hay mucha dispersión en los valores de desigualdad. Sin embargo, observamos niveles de desigualdad relativamente menores en provincias gobernadas por los partidos Socialista, FPV y en menor medida los Radicales K, partidos que podríamos llegar a ubicar a la izquierda del resto (ver Gráfico 8).

En una última estrategia empírica para analizar la robustez de los resultados, evaluamos el impacto de variables políticas (porcentaje de votos y bancas del gobernador) y fiscales clave (empleo público y transferencias nacionales) en tres modelos de regresión diferentes (1-3) y en un modelo final (4) en dónde incluimos todas las variables. Como medida resumen de las variables políticas, utilizamos un índice del poder electoral y legislativo del gobernador, que es una medida compuesta de su poder electoral y legislativo ${ }^{18}$. Seleccionamos las variables mencionadas arriba como representativas del grupo de variables políticas y fiscales porque son las más relevantes teóricamente y porque empíricamente son algunas de las más robustas en las correlaciones. Además, presentan una alta correlación con las otras variables dentro de cada grupo (por lo que se las puede considerar «representativas» del tipo de argumento). Utilizamos una serie de variables socioeconómicas de control, similares a las utilizadas en la literatura existente sobre el tema: crecimiento económico nacional, (el logaritmo natural de) población provincial total y el porcentaje de desempleo provincial.

18 El índice de poder electoral y legislativo del gobernador se compone de dos dimensiones principales: a) el poder de los gobernadores en sus distritos (que incluye el apoyo electoral - proporción de votos-_ para el gobernador; si el partido principal en la legislatura es el partido del gobernador, codificado como 1 en caso de que sean los mismos, 0 de lo contrario, y el porcentaje de bancas del partido del gobernador en la legislatura estatal); y b) la influencia de los gobernadores sobre el gobierno federal o cómo están vinculados políticamente los gobernadores (aquí, incluimos una variable dummy para los casos en que los presidentes y gobernadores están en la misma coalición de gobierno; codificados como 1 en caso de que estén políticamente aliados, 0 de lo contrario). El índice es una medida compuesta de todas las medidas y variables dummy mencionadas anteriormente (que contribuyen 0,5 puntos al índice en caso de que estén codificados como 1, para equilibrar el efecto de cada medida). El valor máximo posible es 3; el mínimo es 0. 


\section{Gráfico 8}

Desigualdad, según Tipo de Partido en las Provincias (2003-2011)

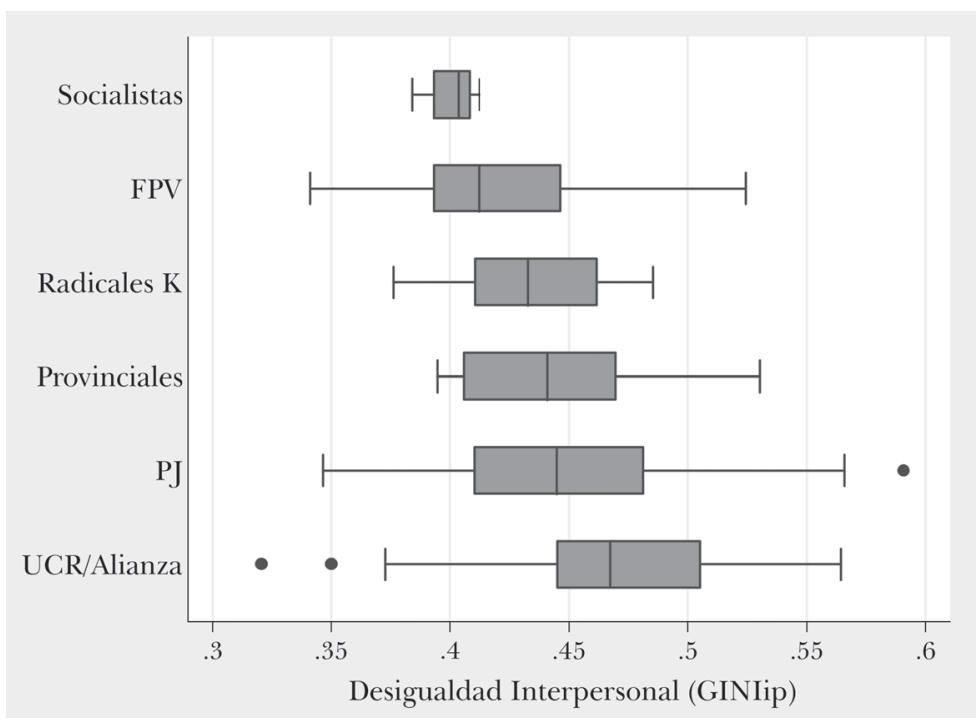

Fuente: elaboración propia.

Debido a la estructura de panel de los datos, algunos supuestos del modelo de regresión lineal (OLS) son problemáticas, especialmente la independencia de las observaciones y de los errores, así como la igual varianza de los errores para todas las observaciones. La prueba de Breusch-Pagan/CookWeisberg y un diagrama de dispersión para el término de error en los modelos principales indican que hay heterocedasticidad. La prueba de Wooldridge indica, además, que hay autocorrelación en los datos del panel. La prueba de Hausman de efectos aleatorios versus efectos fijos reporta un valor de $\mathrm{p}$ en el límite de la significancia estadística para el modelo que usa efectos aleatorios y fijos $(\mathrm{p}=0,1)$. Por lo tanto, se recomienda el uso de un modelo de regresión de efectos fijos. Una forma convencional de lidiar con estos problemas sería utilizar la regresión de mínimos cuadrados ordinarios con errores estándar corregidos para datos de panel (PCSE; Beck y Katz, 1995), para calcular las estimaciones de varianza-covarianza y los errores estándar, asumiendo que las perturbaciones son heterocedásticas y están correlacionadas en los paneles. También incluimos dummies regionales para capturar efectos fijos: una para identificar al Noreste, Noroeste, Cuyo, Patagonia y la Pampa Central (esta es la categoría de referencia). Finalmente, capturamos efectos fijos con variables relativamente invariantes en el tiempo, como la población provincial según el último censo. 


\section{Tabla 4}

Resultados de la Regresión PCSE

\begin{tabular}{|c|c|c|c|c|}
\hline Variables & Modelo 1 & Modelo 2 & Modelo 3 & Modelo 4 \\
\hline $\begin{array}{l}\text { Empleo Público } \\
\text { (log. nat.) }\end{array}$ & $\begin{array}{c}-6.8993 * * * \\
(1.0263)\end{array}$ & -- & -- & $\begin{array}{l}-1.0988 * * \\
(.4819)\end{array}$ \\
\hline $\begin{array}{l}\text { Transferencias Federales } \\
\text { (USD, per capita, log. nat.) }\end{array}$ & -- & $\begin{array}{l}-4.0085^{* * * *} \\
(.3233)\end{array}$ & -- & $\begin{array}{l}-2.4015^{* * *} \\
(.2375)\end{array}$ \\
\hline $\begin{array}{l}\text { Poder Electoral y } \\
\text { Legislativo del Gobernador }\end{array}$ & -- & -- & $\begin{array}{l}-1.6055^{* * * *} \\
(.5536)\end{array}$ & $\begin{array}{l}-2.9879^{*} \\
(1.6268)\end{array}$ \\
\hline $\begin{array}{l}\text { Empleo Agrícola } \\
\text { (\% del empleo total) }\end{array}$ & $\begin{array}{l}.1553 * * * \\
(.0425)\end{array}$ & $\begin{array}{l}.3217 * * * \\
(.0379)\end{array}$ & $\begin{array}{l}.1987 * * * \\
(.0409)\end{array}$ & $\begin{array}{l}.2250 * * * \\
(.0642)\end{array}$ \\
\hline Desempleo & $\begin{array}{l}.7805^{* * *} \\
(.0945)\end{array}$ & $\begin{array}{l}.4241^{* * *} \\
(.0673)\end{array}$ & $\begin{array}{l}.5731^{* * *} \\
(.0754)\end{array}$ & $\begin{array}{l}.4257^{* * *} \\
(.0428)\end{array}$ \\
\hline $\begin{array}{l}\text { Crecimiento Económico } \\
\text { Nacional }\end{array}$ & $\begin{array}{l}.1694 \\
(.1310)\end{array}$ & $\begin{array}{l}.1159^{*} \\
(.0674)\end{array}$ & $\begin{array}{l}.4999 * * * \\
(.0939)\end{array}$ & $\begin{array}{l}.2828^{* * *} \\
(.0750)\end{array}$ \\
\hline $\begin{array}{l}\text { Población Provincial } \\
\text { (log. nat.) }\end{array}$ & $\begin{array}{l}-.8941^{*} \\
(.4587) \\
\end{array}$ & $\begin{array}{l}-1.0756^{* * *} \\
(.2697) \\
\end{array}$ & $\begin{array}{l}-.1591 * \\
(1.4459) \\
\end{array}$ & $\begin{array}{l}-1.0132 * * * \\
(.3855) \\
\end{array}$ \\
\hline Patagonia & $\begin{array}{l}3.5999 * * * \\
(.9594)\end{array}$ & $\begin{array}{l}-.1913 \\
(.9710)\end{array}$ & $\begin{array}{c}-.1591 \\
(1.4459)\end{array}$ & $\begin{array}{c}.6665 \\
(2.1897)\end{array}$ \\
\hline Noreste & $\begin{array}{l}3.8227 * * * \\
(.7802) \\
\end{array}$ & $\begin{array}{l}1.6650^{*} \\
(.8706) \\
\end{array}$ & $\begin{array}{r}2.5602^{*} \\
(1.3332) \\
\end{array}$ & $\begin{array}{l}2.6418^{*} \\
(1.5283) \\
\end{array}$ \\
\hline Noroeste & $\begin{array}{l}4.3429 * * * \\
(.8974)\end{array}$ & $\begin{array}{l}2.5170^{* *} \\
(.9942)\end{array}$ & $\begin{array}{c}2.8454^{*} \\
(1.6160) \\
\end{array}$ & $\begin{array}{l}3.6829 * \\
(2.1013) \\
\end{array}$ \\
\hline Cuyo & $\begin{array}{l}3.1234 * * * \\
(.7204)\end{array}$ & $\begin{array}{l}1.9126^{* *} \\
(.8630)\end{array}$ & $\begin{array}{l}2.9183 * * \\
(1.4122)\end{array}$ & $\begin{array}{l}1.9669 \\
(1.5903)\end{array}$ \\
\hline Constante & $\begin{array}{l}71.8090 * * * \\
(9.7650)\end{array}$ & $\begin{array}{l}76.4759 * * * \\
(5.2320)\end{array}$ & $\begin{array}{l}28.9210^{* * * *} \\
(5.9141)\end{array}$ & $\begin{array}{l}78.2811 \\
(9.1397)\end{array}$ \\
\hline Observaciones & 198 & 198 & 134 & 117 \\
\hline R2 & 0.70 & 0.70 & 0.73 & 0.78 \\
\hline Unidades transversales & 24 & 24 & 24 & 24 \\
\hline
\end{tabular}

Fuente: elaboración propia.

La Tabla 4 muestra los resultados de la regresión entre la desigualdad de ingreso en las provincias y las variables clave en nuestros modelos. Los resultados indican que, controlando por terceras variables, un aumento del uno por ciento en el número de empleados públicos cada 1.000 habitantes y en las transferencias federales per cápita, así como un aumento de un punto en un índice que combina el poder electoral y legislativo del gobernador, producen caídas importantes en el índice de Gini provincial. Esta disminución oscila entre 1.6 y 6.9 puntos promedio (modelos 1-3). En el modelo que combina todas las variables (4), el coeficiente de la variable política aumenta su robustez mientras que las otras dos la disminuyen un poco. Sin embargo, todas ellas conservan su significancia estadística. Las variables socioeconómicas de control, particularmente empleo agrícola y desempleo, 
mantienen el signo esperable, identificado en las correlaciones. La variable crecimiento económico muestra resultados mixtos. Estos modelos explican entre un 70 y un 78 por ciento de la variación de la variable dependiente.

De acuerdo con estos resultados, podemos señalar al menos cinco grandes conclusiones preliminares que pueden servir de base para futuras investigaciones:

Primero, no encontramos evidencia empírica que apoye las expectativas de la TPI en su versión de los márgenes electorales, aunque sí en lo que hace a las reglas electorales. Esto es, el traslado de este marco teórico nacional a la realidad subnacional argentina tiene un sustento empírico sólo parcial, señalando el primer elemento de especificidad subnacional que debe ser indagado tanto teórica como empíricamente.

Segundo, encontramos algún sustento empírico para parte de los argumentos de la TRP, lo que pone al menos algunos interrogantes sobre la muy extendida visión de que la ideología de los partidos y el gobierno (y, correspondientemente, las relaciones entre clases sociales) no son un factor relevante para entender la vida política en el nivel provincial y su relación con variables socioeconómicas.

Tercero, la relación entre los factores políticos y la desigualdad subnacional parece funcionar de manera inversa en la Argentina respecto de los resultados obtenidos en Brasil. Mientras en este país se verifican las previsiones respecto de los márgenes electorales, establecidas por las TPI, en la Argentina estos márgenes operan de modo contrario; y mientras en Brasil los resultados desmienten las expectativas de la TRP, esta parece encontrar cierto respaldo en la Argentina.

Cuarto, cierto número de variables derivadas de teorías «nacionales» («empleo público», «transferencias nacionales», la mayoría de las variables socioeconómicas, además de la ya mencionada «ideología») se vinculan con la desigualdad subnacional en línea con lo establecido por aquellas teorías, lo que implica que, al menos en parte, la escala subnacional reproduce dinámicas y relaciones propias de la escala nacional.

Quinto, no obstante lo anterior, las variables «empleo público»y «años del gobernador en el poder» (no consideradas, al menos hasta ahora, en los estudios nacionales) parecen indicar la existencia de cierta especificidad de los procesos políticos provinciales en su relación con la desigualdad, los cuales, al menos parcialmente, se diferencian de los que son propiamente nacionales. La asunción teórica y metodológica de que las desigualdades subnacionales requieren un abordaje que contemple las particularidades de su escala tiene, en el caso argentino, un preliminar, pero no menos importante, respaldo empírico. 


\section{Palabras Finales}

La desigualdad se distribuye desigualmente entre las unidades subnacionales de los sistemas federales. Esta cuestión es relevante, no sólo por la sobrevivencia de «bolsones» de desigualdad en contextos en los que esta se reduce a nivel nacional, sino también porque los alcances y los límites de las políticas redistributivas nacionales dependen también de lo que sucede en el nivel subnacional, particularmente de los factores políticos propios de este nivel.

Estos factores políticos específicamente subnacionales han sido muy poco explorados por la literatura en el tema en América Latina. Como vimos, los escasos trabajos que se ocupan de la cuestión lo hacen desde una perspectiva anclada teórica y conceptualmente en el nivel nacional.

En este trabajo pretendemos hacer una contribución al desarrollo de esta temática a partir de un examen, exploratorio y preliminar, del caso argentino. Mostramos que las diferencias en los niveles de desigualdad en las provincias argentinas son amplias y se sostienen en un contexto de fuerte reducción de la misma. Por su parte, nuestro análisis, aunque provisorio, muestra indicios de que, además de factores vinculados teóricamente con la escala nacional (como la ideología), los factores específicamente provinciales son relevantes. En este sentido, resalta la incidencia que parece tener el gasto en empleo público, la cantidad de años del partido del gobernador en el poder y los márgenes electorales entre los primeros y segundos partidos sobre la desigualdad en las provincias. En este último caso, la especificidad se manifiesta como un comportamiento de las variables opuesta a la prevista por teorías desarrolladas para la escala nacional.

Estudios futuros podrán profundizar estos resultados preliminares, en primer lugar, ampliando la serie de datos de desigualdad (incluyendo años posteriores a 2011 y años previos al 2003, para compararlos con períodos de aumento en la desigualdad en los 80 s y 90 s). En segundo lugar, precisando mejor las variables provinciales que aparecen como relevantes en este estudio, como posición ideológica de los partidos, tipo de gasto provincial y empleo. En tercer lugar, generando nuevas perspectivas teóricas que capten adecuadamente la relación entre política y desigualdad en la escala subnacional y que especifiquen las diferencias y articulaciones con las teorías disponibles en la escala nacional. En cuarto lugar, avanzando hacia estudios comparativos entre los federalismos latinoamericanos que permitan especificar y explicar tanto las similitudes como las diferencias de estas relaciones entre los casos de estudio. 


\section{Referencias bibliográficas}

Acuña, C. (2013). «Cómo entender las instituciones y su relación con la política: lo bueno, lo malo y lo feo de las instituciones y los institucionalismos» en C. Acuña (comp.), ¿Cuánto importan las instituciones? Gobierno, Estado y actores en la política argentina. Buenos Aires: Siglo XXI

Alderson, A. S. y Nielsen, F. (2002). «Globalization and the great U-turn: Income inequality trends in 16 OECD countries». American Journal of Sociology, 107(5), 1244-1299.

Anderson, C. J. y Beramendi, P. (2012). «Left parties, poor voters, and electoral participation in advanced industrial societies». Comparative Political Studies, 45(6), 714-746.

Ardanaz, M. y Scartascini, C. (2013). «Inequality and personal income taxation: The origins and effects of legislative malapportionment». Comparative Political Studies, 46(12), 1636-1663.

Arretche, Marta. 2009. «Federalism and place-equality policies: a case study of policy design and outputs». European University Institute. Working Papers 2009/ 02.

Austen-Smith, D. (2000). «Redistributing income under proportional representation». Journal of Political Economy, 108(6), 1235-1269.

Avelino, G. (2000). Economic Crisis, Democratization, and Social Spending in Latin America, Unpublished Doctoral Dissertation, Universidad de Stanford, California.

Boix, C. (1998). Political parties, growth and equality: Conservative and social democratic economic strategies in the world economy. New York: Cambridge University Press.

Beramendi, P., M. Rogers y A. Díaz-Cayeros (2017). «Barriers to egalitarianism: distributive tensions in latin american federations». Latin American Research Review, 52(4): 529-551.

Birdsall, N., N. Lustig y D. McLead (2011). «Declining inequality in Latin America: some economics, some politics». Center for Global Development, Working Paper n. 251.

Birchfield, V.y M. Crepaz (1998). «The impact of constitutional structures and collective and competitive veto points on income inequality in industrialized democracies». European Journal of Political Research 34: 175-200

Blofield, M. (2011). «Desigualdad y política en América Latina».Journal of Democracy en Español, 58-74.

Bradley, D., E. Huber, S. Moller, F. Nielsen y J. Stephens (2003). «Distribution and redistribution in postindustrial democracies». World Politics, 55(2), 193-228.

Bueno de Mesquita, B. et al. (2003). The Logic of Political Survival. Cambridge: MIT Press.

Calvo, E. y Moscovich, L. (2017). «Inequality, Protests, and the Progressive Allocation of Cash Transfers in the Argentine Provinces». Latin American Politics and Society, $59(2), 3-26$. 
CEPAL (2017). Panorama Social de América Latina. Documento Informativo. Santiago de Chile: CEPAL.

Chhibber, P. y Nooruddin, I. (2004). «Do party systems count? The Number of Parties and Government Performance in the Indian States». Comparative Political Studies, v. 37, n. 2, 152-187.

Cont, W. y A. Porto (2014). «Personal and regional redistribution through public finance in a federal setting». The Quarterly Review of Economics and Finance, vol. 54, issue 4, 563-578.

Corbacho, A. L. (1998). «Reformas constitucionales y modelos de decisión en la democracia argentina, 1984-1994». Desarrollo Económico, 591-616.

Cornia, G. (2012). «Inequality trends and their determinants: Latin Amercia over 1990-2010». WIDER Working Paper, n 2012/09.

Díaz Cayeros, Alberto (2004). «El federalismo y los límites políticos de la redistribución». Gestión y Política Pública, Vol. XIII, № 3: 663-687.

Esping-Andersen, G. (1990). The Three Worlds of Welfare Capitalism. New Jersey: Princeton University Press.

Giraudy, A. Moncada, E., Snyder, R. (en prensa). Inside Countries: Subnational Research in Comparative Politics. New York: Cambridge University Press.

González, L. y Nazareno, M. (2018). «The Unequal Distribution of Inequality. Subnational Politics and Income Distribution in the Argentine Provinces, 20032011», paper presentado en el workshop del Grupo de Trabajo sobre América Latina (GTAL), Barcelona, Mayo.

González, L.-(2017). «Electoral Competition and Social Spending in the Argentine Provinces». Journal of Politics in Latin America (JPLA), 9 (1), p. 91-124.

González, L. y Cáceres, B. (2017). «Coaliciones Electorales, Estabilidad del Gabinete y Gasto Social Provincial: los Casos de Buenos Aires, Córdoba y San Luis (19932009)». Caderno CRH 30 (80), p. 275-291.

González, L. (2016 a). «Presidential Popularity and the Politics of Distributing Federal Funds in Argentina. Pulibus: The Journal of Federalsim, 46(2), p. 1-25.

González, L. (2016 b). «Ciclo Electoral, Popularidad Presidencial y Distribución de Fondos Federales en Argentina». Revista SAAP, Vol. 10, n. 1, p. 35-64.

González, L. (2014) «Transferencias Federales, Desigualdad Interregional y Redistribución». América Latina Hoy 67, pp.167-190.

Hicks, A. (1999). Social Democracy and Welfare Capitalism: A Century of Income Security Policies. Ithaca: Cornell University Press. 1999.

Hicks, A.; Swank, D. H. (1984). «Redistribution in Rich Capitalist Democracies». Policy Studies Journal, 13(2), p. 265-286.

Huber, E., Stephens, J. D. (2001). Development and crisis of the welfare state: Parties and policies in global markets. University of Chicago press.

Huber E., Stephens, J. D. (2012). Democracy and the Left: Social Policy and Inequality in Latin America. Chicago, IL: University of Chicago Press. 
IPEADATA (2017). Base de datos sobre desigualdade no Brasil. Asesoria Tecnica da Presidencia do Brasil.

Iversen, T. (2001) «The Dynamics of Welfare State Expansion: Trade Openness, DeIndustrialization and Partisan Politics. En Pierson, P. (ed.), The New Politics of the Welfare State. Oxford: Oxford University Press. 2001.

Iversen, T., Soskice, D. (2006). «Electoral institutions and the politics of coalitions: Why some democracies redistribute more than others». American Political Science Review, 100(2), p. 165-181.

Kelly, N. J., Witko, C. (2012). «Federalism and American inequality». The Journal of Politics, 74(2), 414-426.

Kenworthy, L., Pontusson, J. (2005). «Rising inequality and the politics of redistribution in affluent countries». Perspectives on Politics, 3(3), p. 449-471.

Kessler, G. (2014). Controversias sobre la desigualdad. Buenos Aires: Fondo de Cultura Económica.

Korpi, W. (1980). «Social Policy and Distributional Conflict in the Capitalist Democracies: A Preliminary Framework». Western European Politics, 3 (3), 296316.

Lindert, P. H. (1994). «The Rise of Social Spending, 1880-1930». Explorations in Economic History, 31 (1), p. 1-37.

Lindert, P. H. (1996). «What Limits Social Spending». Explorations in Economic History, $33(1), \mathrm{p} 1-34$.

Lindert, P. H. (2004). Growing Public Social Spending and Economic Growth since the Eighteenth Century. Cambridge: Cambridge University Press.

Lupu, N. y Pontusson, J. (2011). «The estructure of inequality and the Politics of redistribution». American Political Science Review, n. 105 (2), p. 316-336.

Lustig, N., L. F. Lopez-Calva y E. Ortiz-Juarez. (2013). «Declining Inequality in Latin America in the 2000s: The Cases of Argentina, Brazil, and Mexico». World Development, 44, p. 129-141.

Magaloni, B., Diaz-Cayeros, A. y Estevez, F. (2004). «Clientelism and Portfolio Diversification: a Model of Electoral Investment with Applications to Mexico». En Kitschelt H. y Wilkinson S., Patrons, Clients, and Policies. Patterns of Democratic Accountability and Political Competition. Cambridge: Cambridge University Press, p. 182-205.

McKay, A. (2002). «Defining and measuring inequality», Inequality Briefing, $\mathrm{N}^{\circ} 1$. UK Department for International Development.

Meltzer, A. H., S. F. Richard. (1981). A Rational Theory of the Size of Government. Journal of Political Economy, 80 (5), p. 914-927.

Moene, K. O., Wallerstein, M. (2001). «Inequality, social insurance, and redistribution». American Political Science Review, 95(4), p. 859-874.

Oszlak, O. y O’Donnell, G. (1976). «Estado y políticas estatales en América Latina: hacia una estrategia de investigación». CEDES, Documento G.E. CLACSO n. 4. 
Pães De Barros, R., Mendonça, R., Rocha, S. (1995). «Brazil: Welfare, Inequality, Poverty, Social Indicators, and Social Programs in the 1980»». En N. Lustig N. (ed), Coping with Austerity. Washington DC: Brookings Institution, p. 237-274.

Persson, T. y Tabellini, G. (2000). Political Economics. Explaining Economic Policy. Cambridge: MIT Press.

Piven, F. y Cloward, R. (1993). Regulating the Poor: The Functions of Public Welfare. New York: Vintage.

Pontusson, J. y Kwon, H. Y. (2003). «Welfare spending, government partisanship, and varieties of capitalism». In delivery at the 2003 Annual Meeting of the American Political Science Association, Philadelphia, PA, pp. 28-31.

Pontusson, J., Rueda, D., Way, C. R. (2002). «Comparative political economy of wage distribution: The role of partisanship and labor market institutions». British Journal of Political Science, 32(2), p. 281-308.

Rodrigues-Silveira, R. (2013). «The subnational method and social policy provision. Socioeconomic context, political institutions and spatial inequality». DesiguALdades.net (Research Network on Interdependent Inequalities in Latina America) Working Papers Series, Working Paper n. 36.

Rodrigues-Silveira, R. (2009). «Federalismo fiscal, desigualdades regionales y políticas contra la pobreza en Brasil: los antagonismos entre políticas sociales universales y el Programa Bolsa Familia». Ponencia presentada en el XVIII Congress of Latina American Studies Association, Río de Janeiro, Brasil, 11-14 de Junio.

Sátyro, N. (2013). «Política Estadual e Desigualdade: Por que algunos Estados Redistribuem Mais do que Outros?». Dados 56 (3), p. 497-530.

Sartori, G. (1970). «Concept misformation in comparative politics». American Political Science Review, 64 (4), p. 1033-53.

Schneider, B. R., Soskice, D. (2009). «Inequality in developed countries and Latin America: coordinated, liberal and hierarchical systems». Economy and society, 38(1), p. 17-52.

Socio-Economic Database for Latin America and the Caribbean (SEDLAC) (2016) CEDLAS/UNLP y Banco Mundial.

Stephens, J. (1979). The transition from capitalism to socialism. Londres: Macmillan.

Varetto, C. (2014). «'Sistema o Sistemas de Partidos? El Enfoque de Pluralidad de Sistemas Partidarios aplicado al Caso Argentino (1983-2011)». Revista Mexicana de Análisis Político y Administración Pública, 3 (2), p. 37-70.

Wallerstein, M. (1999). «Wage-setting institutions and pay inequality in advanced industrial societies». American Journal of Political Science, p. 649-680.

Wibbels, E. (2006). «Madison in Baghdad?: Descentralization and federalism in comparative politics». Annual Review of Political Science, 9 (1), p. 165-168

World Income Inequality Database (WIID) (2017). Helsinki: United Nations University. 


\title{
Palabras Clave
}

desigualdad - política - provincias - Argentina - América Latina

\section{Keywords}

inequality - Politics - provinces - Argentina - Latin America

\begin{abstract}
Despite massive income redistributive policies in recent years, inequality across Latin America is still very high. One of the possible explanations for the resilience of inequality can be found at the subnational level. Pockets of very high inequality persist at this level of government, even when national governments implement large scale redistributive policies. This paper investigates which factors at this level of government may help explaining differences in income inequality across provinces in Argentina. It also intends to contribute to understand the limits imposed to national-led inequality reduction policies. After describing the cases using original panel data on income inequality, the paper identifies structural and political factors systematically related to the levels of inequality in the Argentine provinces.
\end{abstract}


Anexo

Variables y Fuentes de Datos

\begin{tabular}{|c|c|c|c|}
\hline Variable & Descripción & Fuente & Años \\
\hline $\begin{array}{l}\text { Desigualdad } \\
\text { interpersonal } \\
\text { (GINIip) }\end{array}$ & $\begin{array}{l}\text { Desigualdad interpersonal (GINIip), } \\
\text { calculada a partir de los datos de la } \\
\text { encuesta sobre el ingreso per cápita para } \\
\text { cada provincia. }\end{array}$ & $\begin{array}{l}\text { Datos de Encuesta Permanente de Hogares } \\
\text { (EPH) del Instituto Nacional de } \\
\text { Estadísticas y Censos (INDEC), Calvo y } \\
\text { Moscovich (2017). }\end{array}$ & $2003-2011$ \\
\hline Votos al gobernador & $\begin{array}{l}\text { Porcentaje de votos del partido del } \\
\text { gobernador. }\end{array}$ & $\begin{array}{l}\text { Datos Electorales, Dirección Nacional } \\
\text { Electoral, Ministerio del Interior. }\end{array}$ & $1983-2015$ \\
\hline $\begin{array}{l}\text { Diferencia con el } \\
\text { Segundo }\end{array}$ & $\begin{array}{l}\text { Diferencia en el porcentaje de votos entre } \\
\text { el partido del gobernador y el segundo } \\
\text { partido en la elección. }\end{array}$ & $\begin{array}{l}\text { Dirección Nacional Electoral, Ministerio } \\
\text { del Interior. }\end{array}$ & $1983-2015$ \\
\hline Años en el poder & $\begin{array}{l}\text { Años del partido del gobernador en el } \\
\text { poder. }\end{array}$ & $\begin{array}{l}\text { Dirección Nacional Electoral, Ministerio } \\
\text { del Interior. }\end{array}$ & $1983-2015$ \\
\hline Tipo de Partido & $\begin{array}{l}\text { Tipo de Partido: PJ, UCR, Socialistas, FPV, } \\
\text { Radicales K, Provinciales. }\end{array}$ & $\begin{array}{l}\text { Elaboración propia en base a datos de la } \\
\text { Dirección Nacional Electoral, Ministerio } \\
\text { del Interior. }\end{array}$ & \\
\hline $\begin{array}{l}\text { Fragmentación } \\
\text { Partidaria }\end{array}$ & $\begin{array}{l}\text { Número efectivo de partidos (NEP) en } \\
\text { términos de votos y bancas en cada } \\
\text { provincia. }\end{array}$ & $\begin{array}{l}\text { Varetto } 2014 \text { en base a la fórmula de } \\
\text { Laakso and Tagepeera (1979). }\end{array}$ & $1983-2011$ \\
\hline Desempleo & Tasa provincial de desempleo. & $\begin{array}{l}\text { Instituto Nacional de Estadísticas y Censos } \\
\text { (INDEC). }\end{array}$ & $2003-2015$ \\
\hline Empleo Agrícola & $\begin{array}{l}\text { Porcent aje de empleo agrícola provincial } \\
\text { sobre el empleo provincial total. }\end{array}$ & $\begin{array}{l}\text { Ministerio de Trabajo, Empleo y Seguridad } \\
\text { Social. }\end{array}$ & $1996-2014$ \\
\hline Pobreza & $\begin{array}{l}\text { Porcentaje de hogares con necesidades } \\
\text { básicas insatisfechas. Datos de censo } \\
\text { (extrapolados para la serie). }\end{array}$ & $\begin{array}{l}\text { Instituto Nacional de Estadísticas y Censos } \\
\text { (INDEC). }\end{array}$ & $1991-2010$ \\
\hline $\begin{array}{l}\text { Gasto Provincial (per } \\
\text { cápita) }\end{array}$ & Gasto provincial en dólares per cápita. & $\begin{array}{l}\text { Dirección Nacional de Coordinación Fiscal } \\
\text { con las Provincias; Ministerio de Economía. }\end{array}$ & $1991-2008$ \\
\hline $\begin{array}{l}\text { Transferencias } \\
\text { Nacionales }\end{array}$ & $\begin{array}{l}\text { Transferencias nacionales totales en } \\
\text { dólares per cápita. }\end{array}$ & $\begin{array}{l}\text { Dirección Nacional de Coordinación Fiscal } \\
\text { con las Provincias; Ministerio de Economía. }\end{array}$ & $1983-2015$ \\
\hline $\begin{array}{l}\text { Déficit Nacionaly } \\
\text { Provincial }\end{array}$ & $\begin{array}{l}\text { Resultado financiero (ingresos totales } \\
\text { menos gastos totales). }\end{array}$ & $\begin{array}{l}\text { Ministerio de Economía y Dirección } \\
\text { Nacional de Coordinación Fiscal con las } \\
\text { Provincias. }\end{array}$ & $1983-2015$ \\
\hline PBG per cápita & $\begin{array}{l}\text { Producto Bruto Geográfico. Datos anuales, } \\
1993 \text { precios constantes, por provincia, per } \\
\text { cápita. }\end{array}$ & $\begin{array}{l}\text { 1983-1992: Consejo Federal de Inversiones } \\
\text { (CFI); 1993-1997: SAREP, IN DEC. 1998- } \\
\text { 2009: Dirección Nacional de Cuentas } \\
\text { Nacionales, INDEC. }\end{array}$ & 1993-2009 \\
\hline Población & $\begin{array}{l}\text { Población en las provincias. Datos del } \\
\text { censo (extrapolados para la serie). }\end{array}$ & $\begin{array}{l}\text { INDEC (Estadísticas vitales. Información } \\
\text { básica. Ministerio de Salud, Secretaría de } \\
\text { Políticas y Regulación Sanitaria. } \\
\text { Subsecretaría de Planificación, Control, } \\
\text { Regulación y Fiscalización. Dirección de } \\
\text { Estadística e Información en Salud. }\end{array}$ & $1983-2010$ \\
\hline $\begin{array}{l}\text { Crecimiento } \\
\text { Económico }\end{array}$ & Tasa anual de crecimiento económico. & Banco Mundial & $1983-2013$ \\
\hline $\begin{array}{l}\text { Balance Fiscal (déficit } \\
\text { o superávit) }\end{array}$ & $\begin{array}{l}\text { Resultado global de las operaciones del } \\
\text { gobierno nacional, como porcentaje de } \\
\text { PBI. }\end{array}$ & CEPAL - CEPALSTAT & $1990-2013$ \\
\hline
\end{tabular}

